\title{
Study on the clinical efficacy of specific phosphodiesterase inhibitor in patients with pulmonary hypertension due to left heart disease
}

\author{
BING HAN $^{1,2}$ and QINGLI WANG ${ }^{3}$
}

\begin{abstract}
${ }^{1}$ The Key Laboratory of Cardiovascular Remodeling and Function Research, Chinese Ministry of Education and Chinese Ministry of Health, The State and Shandong Joint Key Laboratory of Translational Cardiovascular Medicine, Qilu Hospital of Shandong University, Jinan, Shandong 250012; ${ }^{2}$ Intensive Care Unit; ${ }^{3}$ Internal Medicine Cardiovascular Department, The Sixth People's Hospital of Jinan, Jinan, Shandong 250200, P.R. China
\end{abstract}

Received October 16, 2017; Accepted May 24, 2018

DOI: $10.3892 / \mathrm{etm} .2018 .6310$

\begin{abstract}
Pulmonary hypertension due to left heart disease (PH-LHD) is caused by left ventricular (LV) systolic and/or diastolic dysfunction and left heart valve disease. LV diseases lead to left ventricular filling pressure increases, pulmonary venous obstruction and pulmonary venous pressure increases, and thus to secondary PH. Exercise tolerance is lower and fatality rates are higher in patients with PH-LHD than those in subjects with normal pulmonary arterial pressure. In spite of the progress in the study of the mechanisms of PH-LHD in recent years, no specific treatment is currently available. The efficacy and safety of targeted therapies for pulmonary arterial hypertension remain to be fully established. In the present study, PH-LHD patients were treated with milrinone injection. It was concluded that milrinone significantly reduces pulmonary artery systolic pressure (PASP) in patients with PH-LHD, and significantly improves the cardiac structure, cardiac function and biochemical indexes. PASP was significantly correlated with the left atrial diameter, LV end diastolic diameter, $\mathrm{LV}$ ejection fraction, tricuspid annular plane systolic excursion, right ventricular fractional area change, $\mathrm{N}$-terminal pro-B-type natriuretic peptide and hypersensitive $\mathrm{C}$-reactive protein.
\end{abstract}

Correspondence to: Dr Bing Han, Intensive Care Unit, The Sixth People's Hospital of Jinan, 1920 Huiquan Road, Zhangqiu, Jinan, Shandong 250200, P.R. China

E-mail: james_0803@163.com

Key words: specific phosphodiesterase inhibitor, pulmonary hypertension due to left heart disease, left atrial diameter, left ventricular end-diastolic diameter, left ventricular ejection fraction, pulmonary artery systolic pressure, tricuspid annular plane systolic excursion, right ventricular fractional area change, N-terminal pro-B-type natriuretic peptide, hypersensitive C-reactive protein

\section{Introduction}

Clinically common left heart diseases (LHD) that cause pulmonary hypertension $(\mathrm{PH})$ include the following: i) Heart failure: Left ventricular (LV) systolic dysfunction (e.g., dilated cardiomyopathy and ischemic cardiomyopathy) and LV diastolic dysfunction (e.g., hypertension, coronary heart disease, hypertrophic cardiomyopathy); and ii) valvular heart disease and other diseases, including left atrial disease (e.g., left atrial myxoma or thrombosis), as well as certain types of congenital heart disease. Left heart disease is one of the most common causes of PH in the clinic. Data of a previous study suggest that $2 / 3$ of chronic heart failure cases may be associated with PH (1). An estimate of $60 \%$ of patients with severe LV systolic dysfunction and $70 \%$ of patients with isolated LV diastolic dysfunction may have PH (2). The diagnosis of PH due to LHD (PH-LHD) is based on a mean pulmonary artery pressure (mPAP) of $\geq 25 \mathrm{mmHg}$ determined by a right heart catheter in the resting condition, or of $>30 \mathrm{mmHg}$ in the active state. Furthermore, the simultaneous determination of a left atrial pressure and pulmonary capillary wedge pressure (PCWP) of $>15 \mathrm{mmHg}$ is required for the clinical diagnosis. PH-LHD may occur due to an increased LV filling pressure, and with the pulmonary arterial pressure progressively increasing, the pulmonary artery may contract irreversible damage and pulmonary vascular structural and functional remodeling, resulting in right ventricular dysfunction or failure. Although the study of the mechanisms of PH-LHD has achieved certain progress in recent years, no specific treatment is currently available. The efficacy and safety of targeted therapies for pulmonary artery hypertension (PAH) in patients with PH-LHD remain to be determined. As the results of clinical trials of targeted drugs for PH-LHD are mostly negative or neutral, it is required to explore alternative drugs that are effective against PH-LHD and to prove their effectiveness. Milrinone is a commonly used drug for the treatment of heart failure, and has been reported to also have an effect on PH. In 2012, Guo (3) reported that the phenomenon of secondary PH was more common in patients with severe left heart valvular disease compared with other types of PH-LHD. Inhalation of 
milrinone significantly reduces the pulmonary artery pressure and pulmonary vascular resistance (PVR), increases the cardiac index, while not having any significant effect on the patients' heart rate (HR) and systemic vascular resistance; inhalation of milrinone was efficient in reducing the PH and PVR, and its effect was stronger than that on the systemic circulation, reflecting a good selectivity to pulmonary vessels (3). In 2012, $\mathrm{Yu}$ and Shi (4) indicated that milrinone effectively reduces $\mathrm{PH}$, improves oxygenation and is a safe and effective drug for reducing $\mathrm{PH}$ in patients with congenital heart disease. In 2013, a study by Xu et al (5), including 20 patients with moderate $\mathrm{PH}$ after cardiac surgery, indicated that milrinone has a synergistic effect with dopamine on increasing myocardial contractility and cardiac output to decrease pulmonary artery pressure. In 2014, Yao et al (6) reported that milrinone improves the heart function and effectively reduces $\mathrm{PH}$ in patients with $\mathrm{PH}$ due to congenital heart disease following operation of the plateau area; patients with congenital heart disease in the study came from altitude of 2,100-3,800 m; PH formation and progression were accelerated by hypoxia and low air pressure. As a specific phosphodiesterase inhibitor, milrinone inhibits the cleavage of AMP, and increases intracellular concentrations of cAMP and the influx of $\mathrm{Ca}^{2+}$ to increase myocardial contractility. At the same time, milrinone highly selectively increases the concentration of cGMP in the pulmonary vascular smooth muscle via activation of protein kinase $G$, increased opening of potassium ion channels, hyperpolarization of the cell membrane and inhibition of $\mathrm{Ca}^{2+}$ influx, resulting in a decrease in the intracellular $\mathrm{Ca}^{2+}$ concentration, relaxation of smooth muscle cells and vasodilation, and reduction of pulmonary arterial pressure. In the present study, PH-LHD patients were treated with milrinone injection, and differences in ultrasound indexes and biochemical indexes were observed and analyzed prior to and following treatment to evaluate its efficacy and safety. The innovation of the present study lies in the following points: i) Single primary disease. The cases included in the present study are of coronary heart disease, but previous studies have assessed cases with multiple primary diseases. Due to differences in the pathogenesis of different primary diseases, there are also differences in the mechanisms leading to PH. ii) Different doses. To observe the efficacy and safety of milrinone, different doses were administered to patients with PH-LHD. iii) Different severity. The efficacy of milrinone was assessed in patients with different severities of PH-LHD. iv) Evaluation of right ventricular function. Only few previous studies have assessed the effects of milrinone on right ventricular function in patients with PH-LHD.

\section{Patients and methods}

Patients. A total of 60 coronary heart disease patients with PH-LHD hospitalized between May 2016 and May 2017 were enrolled in the present study. The patients were randomly divided into three groups: Group A and group B as the treatment groups, and group $\mathrm{C}$ as the control group $(\mathrm{n}=20$ in each group). Among them, group A comprised 9 male and 11 female cases (age, 60.2 \pm 3.3 years), group B included 12 male and 8 female cases (age, $59.9 \pm 4.2$ years) and group $C$ comprised 10 male and 10 female cases (age, $60.8 \pm 6.0$ years). There was no significant difference between the three groups in terms of sex, age, supine systolic pressure, supine diastolic pressure, mean arterial pressure and supine HR. All of the participants of the present study provided written informed consent and volunteered to join the study. The study was approved by the Ethics Committee of the Sixth People's Hospital of Jinan (Jinan, China).

Inclusion criteria. The criteria for inclusion were systolic heart failure (left heart failure or total heart failure), LV ejection fraction (LVEF) $\leq 50 \%$. Referring to the 2009 European guidelines for Pulmonary Hypertension, PASP $<37 \mathrm{mmHg}$ under resting condition in normal subjects (7). If PASP is $>50 \mathrm{mmHg}$, it can be used to diagnose PH (7). When $37<\mathrm{PASP}<50 \mathrm{mmHg}$, further cardiac catheterization should be performed for diagnosis (7). In the current study, PASP $>50 \mathrm{mmHg}$ was used as diagnostic criteria for PH-LHD. According to the severity of pulmonary hypertension, patients in each group were divided into a mild group (PASP, 51-60 $\mathrm{mmHg}$ ), a moderate group (PASP, $61-70 \mathrm{mmHg}$ ) and a severe group (PASP, $>70 \mathrm{mmHg}$ ).

Exclusion criteria. i) PH associated with chronic obstructive pulmonary disease, interstitial lung disease, pulmonary disease and hypoxemia caused by sleep apnea, as well as idiopathic, familial and other underlying factors (collagen, portal hypertension, infection, drugs/toxins), and PH caused by chronic thrombosis, embolism or diseases including sarcoidosis and histiocytosis. ii) Acute myocardial infarction, severe arrhythmia, dilated cardiomyopathy, hypertrophic cardiomyopathy, restrictive cardiomyopathy, valvular heart disease or constrictive pericarditis. iii) Cardiogenic shock with insufficient blood volume or vasodilator. iv) Supine systolic pressure $<90$ or $>180 \mathrm{mmHg}$. v) Pulmonary vascular malformation. vi) Cerebrovascular accident. vii) Malignant tumor. viii) Severe hepatic dysfunction (elevated transaminase exceeding the normal limit by 3 -fold) and renal failure. ix) Serious infectious diseases. $x$ ) Uncontrolled thyroid disease. xi) Cyclophilin drugs and endothelin receptor antagonists have been used in the past and other targeted drugs for pulmonary hypertension. xii) Participation in other clinical studies in the previous 3 months. xiii) A history of allergies to specific phosphodiesterase inhibitors or contraindications for the use of such drugs, including hypotension and arrhythmias. xiv) Cardiac resynchronization pacemaker. xv) Mental sickness.

Research drug use. The research drug was milrinone injection, whose commodity name is Lunan Li Kang, produced by Nonan Bate Pharmaceuticals Co., Ltd (Linyi, China; approval no. H10970051). A volume of $5 \mathrm{ml}$ of the formulation was equivalent to $5 \mathrm{mg}$ milrinone.

Equipment and preparations. A Philips CX-50 type color Doppler ultrasound (Philips Medical Co., Eindhoven, the Netherlands) was used in the present study. NT-proBNP was detected by a Roche Cobas-e601 automatic biochemical analyzer (Roche Diagnostics, Basel, Switzerland). Determination of NT-proBNP was performed with a chemiluminescent immunoassay provided by Roche Diagnostics (cat. nos. 14129204, 16899201 and 16899203). HsCRP was detected by a Siemens BN II specific protein 
analyzer (Siemens AG, Munich, Germany). The hsCRP kit was provided by Siemens AG (cat. nos. 167551C and 167553C).

Treatment. The same conventional treatment was used in the three groups, including digitalis, angiotensin-converting enzyme inhibitor/angiotensin II receptor antagonist, $\beta$ receptor blocker and diuretics. First, group A and group B were given intravenous milrinone for $10 \mathrm{~min}$ at rate $5 \mu \mathrm{g} / \mathrm{kg} / \mathrm{min}$. The maintenance dose for group A and B were 0.25 and $0.5 \mu \mathrm{g} / \mathrm{kg} / \mathrm{min}$ of milrinone, respectively, administered by continuous application for 5 days. Group $\mathrm{C}$ was not treated with milrinone. Echocardiography was performed prior and following milrinone treatment and blood samples were taken to determine the biochemical parameters. $\mathrm{HR}$, blood pressure (BP) and various examination results were monitored prior to and following treatment.

\section{Observation indexes}

General observation indexes. The following indicators were determined prior to treatment: $\mathrm{BP}, \mathrm{HR}$, blood routine, urine routine, stool routine, liver function, renal function, blood glucose, blood lipids, blood electrolytes and electrocardiogram examination.

Echocardiography. Echocardiography was performed prior and following milrinone treatment. The ultrasonic instrument was a Philips CX-50 type color Doppler ultrasound, equipped with a S51 heart two-dimensional probe, a frequency of 1-5 MHz and a frame frequency of 60-90 frames/s. The patient was placed in the left lateral decubitus position. M-mode echocardiography was used on the long axial of sternum side to measure the left atrial diameter (LAD), LV end diastolic diameter (LVEDd) and LVEF. The apical four chamber was viewed and the tricuspid annular plane systolic excursion (TAPSE) was measured. The right ventricular end-diastolic area and end-systole area was measured, and the right ventricular fractional area change (RVFAC) was calculated. PASP was measured by tricuspid regurgitation estimation, with PASP equaling the right ventricular systolic pressure (RVSP). The RVSP was calculated according to the modified formula of Bernoulli (RVSP $=4 \mathrm{Vmax}^{2}+\mathrm{RAP}$ ), where Vmax is the maximum tricuspid regurgitation velocity $(\mathrm{m} / \mathrm{sec})$ and RAP is the right atrial pressure (8). The RAP may be estimated based on the size of the right atrium and the extent of the tricuspid regurgitation. The right atrium diameter was normal, mild tricuspid regurgitation was observed and the RAP was $5 \mathrm{mmHg}$. The right atrium was slightly enlarged, moderate three tricuspid regurgitation occurred and the RAP was $10 \mathrm{mmHg}$. The right atrium was markedly enlarged, severe three tricuspid regurgitation was observed and the RAP was $15 \mathrm{mmHg}$. The above indicators were measured by two ultrasound attending physicians with 5 years of experience who were blinded to the grouping. All records were stored on a computer for data analysis.

Detection of NT-proBNP. Blood $(5 \mathrm{ml})$ was drawn from the elbow vein prior to and following pre-therapy, collected in EDTA anticoagulant vacuum tubes, and after standing for $30 \mathrm{~min}$ at room temperature, serum was separated by centrifugation at $1,000 \mathrm{xg}$ for $15 \mathrm{~min}$ at room temperature. The concentration of NT-proBNP was determined with a
Roche Cobas-e601 automatic biochemical analyzer with an electrochemiluminescence immunoassay. The range of measurements covered by the assay was 5-35,000 $\mathrm{pg} / \mathrm{ml}$. The determination principle is the double antibody clamp method and the protocol was as follows: First, $20 \mu \mathrm{l}$ specimen, biotinylated monoclonal NT-proBNP-specific antibody and monoclonal NT-proBNP-specific antibody labeled with ruthenium complex were reacted to form a Sandwich complex. Subsequently, magnetic beads coated with streptavidin were added, and through the interaction between biotin and streptavidin, the sandwich complex was bound to the magnetic beads. The reaction mixture was added to a measuring tank, where magnetic particles were adsorbed to the electrode surface by electromagnetic action. Voltage was added to the electrode to produce electrochemiluminescence and light signals were measured using photomultiplier tubes. Specific curves were obtained by two-point calibration and the major curve provided by the reagent bar code was used to determine the results.

Detection of hsCRP. The concentration of hsCRP in the serum was determined by a Siemens BN II specific protein analyzer via an immunoturbidimetric test (normal value, $0-3 \mathrm{mg} / \mathrm{ml}$ ). Its determination principle was as follows: The CRP in the sample is reacted with the CRP-specific antibody coated on latex particles contained in the reagent to form an immunoprecipitated, whose amount is proportional to the concentration of CRP in the specimen.

Observation of adverse events. By clinical observation, type, time, duration, severity, process and outcome of adverse events that occurred following administration were recorded. The safety of the drug was evaluated objectively by recording whether the drug administration was terminated and whether any measures to treat the adverse events were taken. The adverse events were then classified into five levels of relevance: Definitely relevant, probably relevant, possibly relevant, possibly irrelevant and irrelevant. The items in the first three levels were classified as adverse reactions and their incidence was determined. Once an adverse reaction occurred, the necessary treatment measures were taken according to the condition, which included adjustment of the dose, temporary interruption of drug use and decision to terminate the administration. Patients with severe adverse reactions were discontinued.

Statistical analysis. In the present study, SPSS 22.0 statistical software (IBM Corp., Armonk, NY, USA) was applied for statistical analysis. The measurement data were expressed as the mean \pm standard deviation. The differences in measurement data among the groups were analyzed by a homogeneity test of variances (least-significant difference method). Analysis of variance followed by Tukey's honest significant differences test was applied to make pairwise comparisons. For the above, $\mathrm{P}<0.05$ was considered to indicate a statistically significant difference. The paired t-test was used to compare differences between the pre-therapy and post-treatment groups, and $\mathrm{P}<0.05$ was considered to indicate a statistically significant difference. Pearson's correlation coefficient was determined to assess the correlation between PASP and LAD, LVEDd, LVEF, TAPSE, 
Table I. Comparison of general data, echocardiography results and biochemical markers levels of three groups of patients with pulmonary hypertension due to left heart disease prior to treatment.

\begin{tabular}{lccccr}
\hline Parameter & Group A $(\mathrm{n}=20)$ & Group B $(\mathrm{n}=20)$ & Group C $(\mathrm{n}=20)$ & F & P-value \\
\hline Age (years) & $60.2 \pm 3.3$ & $59.9 \pm 4.2$ & $60.8 \pm 6.0$ & 0.035 & 0.965 \\
Male sex [cases (\%)] & $9(45)$ & $12(60)$ & $10(50)$ & 1.262 & 0.291 \\
SBP (mmHg) & $132.2 \pm 4.8$ & $133.8 \pm 9.1$ & $128.2 \pm 8.0$ & 0.039 & 0.962 \\
NBP (mmHg) & $86.8 \pm 5.2$ & $84.7 \pm 5.4$ & $85.3 \pm 4.0$ & 0.053 & 0.948 \\
MAP (mmHg) & $101.9 \pm 4.7$ & $101.4 \pm 6.4$ & $99.6 \pm 5.1$ & 0.034 & 0.967 \\
HR (per min) & $80.6 \pm 10.3$ & $83.6 \pm 8.0$ & $81.1 \pm 8.9$ & 0.042 & 0.959 \\
LAD (mm) & $46.4 \pm 4.1$ & $46.5 \pm 4.8$ & $46.2 \pm 4.7$ & 0.015 & 0.985 \\
LVEDd (mm) & $61.7 \pm 5.4$ & $62.0 \pm 6.6$ & $61.9 \pm 4.6$ & 0.015 & 0.985 \\
LVEF (\%) & $39.1 \pm 6.2$ & $39.6 \pm 7.3$ & $38.1 \pm 6.1$ & 0.269 & 0.765 \\
PASP (mmHg) & $62.3 \pm 6.9$ & $62.1 \pm 6.3$ & $62.4 \pm 7.4$ & 0.009 & 0.991 \\
TAPSE (mm) & $15.0 \pm 3.6$ & $15.1 \pm 3.4$ & $15.1 \pm 2.8$ & 0.011 & 0.989 \\
RVFAC (\%) & $35.1 \pm 4.1$ & $35.2 \pm 4.2$ & $35.0 \pm 4.7$ & 0.011 & 0.989 \\
NT-proBNP (pg/ml) & $5,153.4 \pm 1,821.2$ & $5,176.3 \pm 1,675.4$ & $5,157.3 \pm 1,924.5$ & 0.001 & 0.999 \\
hsCRP (mg/l) & $6.5 \pm 2.9$ & $6.3 \pm 3.6$ & $5.5 \pm 2.9$ & 0.566 & 0.571 \\
\hline
\end{tabular}

Data are presented as the mean \pm standard deviation or $\mathrm{n}(\%)$. LAD, left atrial diameter; LVEDd, left ventricle end diastolic diameter; LVEF, left ventricle ejection fraction; PASP, pulmonary artery systolic pressure; TAPSE, tricuspid annular plane systolic excursion; RVFAC, right ventricular fractional area change; NT-proBNP, N-terminal pro-B-type natriuretic peptide; hsCRP, hypersensitive C-reactive protein; HR, heart rate; SBP, systolic blood pressure; MAP, mean arterial pressure; NBP, noninvasive blood pressure.

RVFAC, NT-proBNP and hsCRP, with $0.8<\mathrm{x} \leq 1.0$, extremely strong correlation; $0.6<\mathrm{x} \leq 0.8$, strong correlation; $0.4<\mathrm{x} \leq 0.6$, medium correlation; $0.2<\mathrm{x} \leq 0.4$, weak correlation; $0.0-0.2$, very weak or no correlation. $\mathrm{P}<0.01$ was considered to indicate a statistically significant difference.

\section{Results}

Experimental results prior to treatment. The treatment groups (groups A and B) and the control group C exhibited no statistically significant differences in the measurement indexes (LAD, LVEDd, LVEF, PASP, TAPSE, RVFAC, NT-proBNP and hsCRP) prior to treatment $(\mathrm{P}>0.05$; Table I).

Comparison of echocardiographic results and levels of biochemical markers between the three groups of PH-LHD patients prior to and following treatment. In group $\mathrm{A}$, the RVFAC following treatment was significantly higher compared with prior to treatment $(\mathrm{P}<0.01$; Table II). The LVEF and TAPSE following treatment were significantly higher compared with prior to treatment $(\mathrm{P}<0.05$; Table II). The LVEDd, PASP, NT-proBNP and hsCRP following treatment were significantly lower compared with prior to treatment $(\mathrm{P}<0.01$; Table II). The LAD following treatment was significantly lower compared with prior to treatment $(\mathrm{P}<0.05$; Table II).

In group $\mathrm{B}$, the LVEF, TAPSE and RVFAC following treatment were higher than those prior to treatment, and the difference was statistically significant $(\mathrm{P}<0.01$; Table II). The LAD, LVEDd, PASP, NT-proBNP and hsCRP following treatment were lower than those prior to treatment, and the difference was statistically significant $(\mathrm{P}<0.01$; Table II).

In group $\mathrm{C}$, the NT-proBNP following treatment was lower than that prior to treatment, and the difference was statistically significant $(\mathrm{P}<0.01$; Table II). The LVEF, TAPSE and RVFAC following treatment were higher than those prior to treatment, the difference was not statistically significant $(\mathrm{P}>0.05$; Table II). The LAD, LVEDd, PASP and hsCRP following treatment were lower than those prior to treatment, the difference was not statistically significant $(\mathrm{P}>0.05$; Table II).

Comparison of echocardiography results and levels of biochemical markers in patients of the different groups following treatment. Following treatment, the LVEF in group $\mathrm{B}$ was higher compared with that in group $\mathrm{A}$, the difference was not statistically significant ( $P>0.05$; Table III). The TAPSE and RVFAC of group B were higher than those of group A, and the difference was statistically significant $(\mathrm{P}<0.05$; Table III). The PASP of group B was lower than that of group A, and the difference was statistically significant $(\mathrm{P}<0.05$; Table III). The LAD, LVEDd, NT-proBNP and hsCRP of group B were lower than those of group $\mathrm{A}$; the difference was not statistically significant $(\mathrm{P}>0.05$; Table III).

Following treatment, the TAPSE and RVFAC of group A were higher than those of group $\mathrm{C}$, and the difference was statistically significant $(\mathrm{P}<0.05$; Table III). The PASP, NT-proBNP and hsCRP of group A were lower than those of group $\mathrm{C}$, and the difference was statistically significant $(\mathrm{P}<0.05$; Table III). The LVEF of group A was higher than that of group $C$; the difference was not statistically significant (P>0.05; Table III). The LAD and LVEDd of group A were lower than those of group $\mathrm{C}$; the difference was not statistically significant $(\mathrm{P}>0.05$; Table III).

Following treatment, the LVEF, TAPSE and RVFAC of group B were higher than those of group $\mathrm{C}$, and the difference was statistically significant $(\mathrm{P}<0.05$; Table III). The LAD, LVEDd, PASP, NT-proBNP and hsCRP of group B were lower 
than those of group $\mathrm{C}$, and the difference was also statistically significant $(\mathrm{P}<0.05$; Table III).

Comparison of echocardiographic results and biochemical markers levels between patients with different severities of $P H-L H D$ prior to and following treatment. In group A, the LVEF, TAPSE and RVFAC in patients with mild $(\mathrm{n}=10)$ and moderate $(n=8)$ PH-LHD following treatment were higher than those prior to treatment, and the difference was statistically significant ( $\mathrm{P}<0.01$; Table IV). The LAD, LVEDd, PASP, NT-proBNP and hsCRP in patients with mild and moderate PH-LHD following treatment were lower than those prior to treatment, and the difference was statistically significant $(\mathrm{P}<0.01$; Table IV $)$.

In group B, the LVEF, TAPSE and RVFAC in patients with mild $(n=10)$ and moderate $(n=8)$ PH-LHD following treatment were higher than those prior to treatment, and the difference was statistically significant $(\mathrm{P}<0.01$; Table $\mathrm{V})$. The LAD, LVEDd, PASP, NT-proBNP and hsCRP in the patients with mild and moderate PH-LHD following treatment were lower than those prior to treatment, and the difference was statistically significant $(\mathrm{P}<0.01$; Table $\mathrm{V})$.

In group $\mathrm{C}$, the RVFAC in patients with mild $(\mathrm{n}=11)$ and moderate $(\mathrm{n}=8) \mathrm{PH}-\mathrm{LHD}$ following treatment was higher than that prior to treatment and the difference was statistically significant $(\mathrm{P}<0.01$; Table VI). The TAPSE in patients with mild PH-LHD following treatment was higher than that prior to treatment, and the difference was statistically significant $(\mathrm{P}<0.05$; Table VI). The TAPSE in patients with moderate PH-LHD following treatment was higher than that prior to treatment, and the difference was not statistically significant $(\mathrm{P}>0.05$; Table VI). The LVEF in patients with mild and moderate PH-LHD following treatment was higher than that prior to treatment, and the difference was statistically significant $(\mathrm{P}<0.01$ and $\mathrm{P}<0.05$; Table VI). The LAD, LVEDd, PASP and NT-proBNP in the patients with mild and moderate PH-LHD following treatment were lower than those prior to treatment, and the difference was statistically significant $(\mathrm{P}<0.01$; Table VI). The hsCRP in the patients with mild PH-LHD following treatment was lower than that prior to treatment, and the difference was statistically significant $(\mathrm{P}<0.01$; Table VI). The hsCRP in the patients with moderate PH-LHD following treatment was lower than that prior to treatment, and the difference was not statistically significant ( $\mathrm{P}>0.05$; Table VI).

Correlation between PASP and other indicators. PASP was positively correlated with LAD, LVEDd, NT-proBNP and hsCRP $(\mathrm{P}<0.01$; Fig. 1; Table VII), and negatively correlated with LVEF, TAPSE and RVFAC (P<0.01; Fig. 2; Table VII).

Comparison of adverse reactions in the three groups of patients with $P H-L H D$. In group A ( $\mathrm{n}=20), 2$ cases of headache (10\%), 2 cases of asthenia (10\%), 1 case of HR increase (5\%) and 1 case of hypotension (5\%) were encountered. Group B $(\mathrm{n}=20)$ included 1 case of headache (5\%), 2 cases of asthenia (10\%), 1 case of HR increase (5\%) and 3 cases of hypotension $(15 \%)$. In group $\mathrm{C}(\mathrm{n}=20), 1$ case of headache $(5 \%), 1$ case of asthenia (5\%) and 1 cases of HR increase $(5 \%)$ were encountered (Table VIII). The maintenance dose of milrinone 
Table III. Comparison of echocardiography results and levels of biochemical markers between the three groups following treatment.

\begin{tabular}{lccccccccc}
\hline & & & & & & & \multicolumn{2}{c}{ ANOVA } \\
\cline { 6 - 10 } Parameter & Group A $(\mathrm{n}=20)$ & Group B $(\mathrm{n}=20)$ & Group C $(\mathrm{n}=20)$ & P1 & P2 & P3 & F & P \\
\hline LAD (mm) & $43.0 \pm 4.9$ & $39.7 \pm 5.9$ & $44.5 \pm 5.5$ & 0.061 & 0.012 & 0.381 & 4.032 & 0.023 \\
LVEDd (mm) & $56.7 \pm 4.7$ & $54.5 \pm 5.6$ & $59.7 \pm 5.0$ & 0.187 & 0.014 & 0.154 & 3.712 & 0.031 \\
LVEF (\%) & $44.0 \pm 6.6$ & $49.4 \pm 8.2$ & $40.4 \pm 6.3$ & 0.064 & 0.001 & 0.112 & 6.195 & 0.004 \\
PASP (mmHg) & $52.9 \pm 7.4$ & $47.3 \pm 7.6$ & $59.4 \pm 8.7$ & 0.025 & 0.000 & 0.015 & 11.547 & 0.000 \\
TAPSE (mm) & $17.4 \pm 4.0$ & $18.4 \pm 4.0$ & $15.8 \pm 3.2$ & 0.037 & 0.000 & 0.043 & 9.119 & 0.000 \\
RVFAC $(\%)$ & $40.7 \pm 5.0$ & $44.2 \pm 5.7$ & $36.9 \pm 4.9$ & 0.046 & 0.000 & 0.022 & 9.663 & 0.000 \\
NT-proBNP (pg/ml) & $3,526.6 \pm 1,333.3$ & $2,863.2 \pm 1,098.8$ & $4,581.0 \pm 1,850.8$ & 0.094 & 0.001 & 0.046 & 7.024 & 0.000 \\
hsCRP (mg/l) & $3.3 \pm 1.8$ & $2.8 \pm 1.6$ & $5.1 \pm 3.0$ & 0.375 & 0.005 & 0.029 & 5.768 & 0.005 \\
\hline
\end{tabular}

Values are expressed as the mean \pm standard deviation. P1, group A vs. B; P2, group B vs. C; P3, group A vs. C. P, P-value; LAD, left atrial diameter; LVEDd, left ventricle end diastolic diameter; LVEF, left ventricle ejection fraction; PASP, pulmonary artery systolic pressure; TAPSE, tricuspid annular plane systolic excursion; RVFAC, right ventricular fractional area change; NT-proBNP, N-terminal pro-B-type natriuretic peptide; hsCRP, hypersensitive C-reactive protein; ANOVA, analysis of variance.

Table IV. Comparison of echocardiographic results and levels of biochemical markers between patients with different severities of PH due to left heart disease in group A prior to and following treatment.

\begin{tabular}{|c|c|c|c|c|c|c|}
\hline \multirow[b]{2}{*}{ Parameter } & \multicolumn{3}{|c|}{ Mild PH (n=10) } & \multicolumn{3}{|c|}{ Moderate PH $(\mathrm{n}=8)$} \\
\hline & Pre-treatment & Post-treatment & P-value & Pre-treatment & Post-treatment & $\mathrm{P}$-value \\
\hline $\mathrm{LAD}(\mathrm{mm})$ & $43.5 \pm 3.0$ & $39.5 \pm 3.5$ & $<0.001$ & $48.0 \pm 1.3$ & $45.1 \pm 1.5$ & $<0.001$ \\
\hline LVEDd (mm) & $58.2 \pm 3.4$ & $53.6 \pm 2.4$ & $<0.001$ & $63.4 \pm 3.3$ & $58.1 \pm 2.6$ & $<0.001$ \\
\hline $\operatorname{LVEF}(\%)$ & $44.1 \pm 2.8$ & $49.1 \pm 3.1$ & $<0.001$ & $35.8 \pm 2.6$ & $40.6 \pm 3.3$ & $<0.001$ \\
\hline PASP $(\mathrm{mmHg})$ & $56.5 \pm 2.6$ & $46.8 \pm 3.6$ & $<0.001$ & $66.0 \pm 2.1$ & $56.9 \pm 2.0$ & $<0.001$ \\
\hline TAPSE (mm) & $17.4 \pm 1.4$ & $21.2 \pm 1.8$ & $<0.001$ & $14.4 \pm 1.7$ & $18.0 \pm 3.1$ & $<0.001$ \\
\hline RVFAC (\%) & $38.1 \pm 2.0$ & $46.5 \pm 2.5$ & $<0.001$ & $33.7 \pm 2.9$ & $40.6 \pm 3.5$ & $<0.001$ \\
\hline NT-proBNP (pg/ml) & $3,918.6 \pm 283.3$ & $2,580.0 \pm 104.8$ & $<0.001$ & $5,622.9 \pm 681.9$ & $3,903.5 \pm 603.9$ & $<0.001$ \\
\hline hsCRP (mg/l) & $4.4 \pm 1.0$ & $1.9 \pm 0.5$ & $<0.001$ & $7.3 \pm 0.9$ & $3.95 \pm 0.7$ & $<0.001$ \\
\hline
\end{tabular}

Values are expressed as the mean \pm standard deviation. LAD, left atrial diameter; LVEDd, left ventricle end diastolic diameter; LVEF, left ventricle ejection fraction; PASP, pulmonary artery systolic pressure; TAPSE, tricuspid annular plane systolic excursion; RVFAC, right ventricular fractional area change; NT-proBNP, N-terminal pro-B-type natriuretic peptide; hsCRP, hypersensitive C-reactive protein; PH, pulmonary hypertension.

did not cause any significant adverse reactions during the course of treatment.

\section{Discussion}

A retrospective review from the US indicated that $77 \%$ of the centers used PAH targeted drugs for patients with PH-LHD (9). Clinical trials on targeted drugs for PH mostly provided negative or neutral results, and the choice of the test population had a decisive role in the outcome, it is also possible that the effective dose of PAH and PH-LHD is different. Furthermore, the mechanism of pulmonary vascular remodeling in PH-LHD requires further elucidation. In 2015, a study by Li et al (10) reported on the efficacy of the combined use of atorvastatin and sodium ferulate in the treatment to PH-LHD based on routine treatment and the safety was good, the PASP decreased and the Tei index (ratio of isovolumic contraction time and isovolumic relaxation time to ejection time.) of the right ventricle decreased $(\mathrm{P}<0.05)$. In 2013, Lian et al (11) indicated that the LVEF was increased in patients with chronic heart failure and PH treated with levosimendan combined with sildenafil compared with that in the control group; the PAH decreased and the 6-min walking distance improved.

Milrinone is an inhibitor of phosphodiesterase III (PDE III). PDE III is distributed and dominant in pulmonary airway smooth muscle, vascular smooth muscle and endothelial cells. Milrinone is highly selective in inhibiting PDE III in myocardial cells and vascular smooth muscle cells, reduces the degradation of cAMP, increases the levels of cAMP in 
Table V. Comparison of echocardiographic results and levels of biochemical markers between patients with different severities of PH due to left heart disease in group B prior to and following treatment.

\begin{tabular}{|c|c|c|c|c|c|c|}
\hline \multirow[b]{2}{*}{ Parameter } & \multicolumn{3}{|c|}{ Mild PH (n=10) } & \multicolumn{3}{|c|}{ Moderate PH (n=8) } \\
\hline & Pre-treatment & Post-treatment & P-value & Pre-treatment & Post-treatment & P-value \\
\hline LAD (mm) & $43.2 \pm 4.0$ & $35.6 \pm 4.1$ & $<0.001$ & $47.7 \pm 1.4$ & $41.2 \pm 2.4$ & $<0.001$ \\
\hline LVEDd (mm) & $58.3 \pm 2.6$ & $51.0 \pm 1.7$ & $<0.001$ & $62.4 \pm 5.4$ & $55.2 \pm 4.2$ & $<0.001$ \\
\hline LVEF (\%) & $44.7 \pm 2.8$ & $55.0 \pm 3.9$ & $<0.001$ & $38.0 \pm 6.4$ & $47.8 \pm 6.5$ & $<0.001$ \\
\hline PASP (mmHg) & $56.2 \pm 2.9$ & $40.4 \pm 3.3$ & $<0.001$ & $65.6 \pm 1.9$ & $50.8 \pm 0.7$ & $<0.001$ \\
\hline TAPSE (mm) & $17.7 \pm 0.7$ & $25.4 \pm 1.2$ & $<0.001$ & $14.0 \pm 1.3$ & $19.5 \pm 2.6$ & $<0.001$ \\
\hline RVFAC (\%) & $37.7 \pm 2.0$ & $48.4 \pm 1.4$ & $<0.001$ & $33.9 \pm 2.6$ & $42.1 \pm 2.4$ & $<0.001$ \\
\hline NT-proBNP (pg/ml) & $3,911.7 \pm 288.1$ & $2,022.2 \pm 137.9$ & $<0.001$ & $5,576.7 \pm 789.2$ & $3,197.3 \pm 680.1$ & $<0.001$ \\
\hline hsCRP (mg/l) & $3.8 \pm 1.2$ & $1.6 \pm 0.4$ & $<0.001$ & $6.9 \pm 1.5$ & $3.2 \pm 0.6$ & $<0.001$ \\
\hline
\end{tabular}

Values are expressed as the mean \pm standard deviation. LAD, left atrial diameter; LVEDd, left ventricle end diastolic diameter; LVEF, left ventricle ejection fraction; PASP, pulmonary artery systolic pressure; TAPSE, tricuspid annular plane systolic excursion; RVFAC, right ventricular fractional area change; NT-proBNP, N-terminal pro-B-type natriuretic peptide; hsCRP, hypersensitive C-reactive protein; $\mathrm{PH}$, pulmonary hypertension.

Table VI. Comparison of echocardiographic results and levels of biochemical markers between patients with different severities $\mathrm{PH}$ due to left heart disease in group $\mathrm{C}$ prior to and following treatment.

\begin{tabular}{|c|c|c|c|c|c|c|}
\hline \multirow[b]{2}{*}{ Parameter } & \multicolumn{3}{|c|}{ Mild group PH $(\mathrm{n}=11)$} & \multicolumn{3}{|c|}{ Moderate group PH $(\mathrm{n}=8)$} \\
\hline & Pre-treatment & Post-treatment & P-value & Pre-treatment & Post-treatment & P-value \\
\hline $\mathrm{LAD}(\mathrm{mm})$ & $43.0 \pm 3.2$ & $40.8 \pm 3.7$ & $<0.001$ & $49.4 \pm 2.2$ & $48.0 \pm 2.7$ & 0.001 \\
\hline LVEDd (mm) & $58.8 \pm 2.6$ & $56.3 \pm 2.4$ & $<0.001$ & $64.6 \pm 2.4$ & $62.8 \pm 2.4$ & $<0.001$ \\
\hline LVEF (\%) & $42.6 \pm 1.7$ & $44.6 \pm 2.4$ & 0.001 & $33.9 \pm 2.9$ & $36.6 \pm 4.1$ & 0.019 \\
\hline PASP $(\mathrm{mmHg})$ & $56.8 \pm 2.3$ & $52.8 \pm 3.8$ & $<0.001$ & $67.5 \pm 1.9$ & $65.5 \pm 1.9$ & $<0.001$ \\
\hline TAPSE (mm) & $16.8 \pm 0.8$ & $17.6 \pm 0.9$ & 0.011 & $13.4 \pm 2.1$ & $13.9 \pm 2.7$ & 0.170 \\
\hline RVFAC (\%) & $38.7 \pm 1.3$ & $40.6 \pm 1.3$ & $<0.001$ & $31.3 \pm 1.5$ & $33.3 \pm 2.3$ & 0.001 \\
\hline NT-proBNP (pg/ml) & $4,008.5 \pm 183.0$ & $3,525.5 \pm 182.0$ & $<0.001$ & $5,908.5 \pm 1,017.4$ & $5,223.4 \pm 1,024.0$ & $<0.001$ \\
\hline $\mathrm{hsCRP}(\mathrm{mg} / \mathrm{l})$ & $3.3 \pm 0.6$ & $2.8 \pm 0.8$ & 0.003 & $7.7 \pm 1.9$ & $7.4 \pm 1.8$ & 0.186 \\
\hline
\end{tabular}

Values are expressed as the mean \pm standard deviation. LAD, left atrial diameter; LVEDd, left ventricle end diastolic diameter; LVEF, left ventricle ejection fraction; PASP, pulmonary artery systolic pressure; TAPSE, tricuspid annular plane systolic excursion; RVFAC, right ventricular fractional area change; NT-proBNP, N-terminal pro-B-type natriuretic peptide; hsCRP, hypersensitive C-reactive protein; PH, pulmonary hypertension.

cardiac myocytes, acts on a series of intracellular protein kinases, opens the calcium channel on the sarcoplasmic reticulum causing large amounts of calcium ions to enter the cytoplasm, and improves the contractility of myocardium by stimulating contraction coupling mechanisms. Milrinone increases the level of cGMP in vascular smooth muscle cells, inhibits the phosphorylation of myosin light chain kinase and calcium reabsorption in the sarcoplasmic reticulum, resulting in a decrease in cytosolic calcium levels, while the contraction and coupling process of smooth muscle is inhibited, finally leading to arterial and venous dilatation (12). Therefore, milrinone increases myocardial contractility and reduces the ventricular load. Various experimental studies have indicated that milrinone reduces the concentration of inflammatory factors, reduces the adhesion between inflammatory cells and vascular endothelial cells, and also reduces the damage caused by the inflammatory response to vascular endothelium by increasing the intracellular concentration of cAMP (13-17). Milrinone antagonizes intracellular calcium overload and maintains tight junctions between endothelial and stromal cells. Activation of cyclase and restrainment of phosphodiesterase may reduce the gap formation between cells and decrease vascular permeability (18). Milrinone has a short plasma half-life, and $80 \%$ of the drug is excreted by the kidneys within $24 \mathrm{~h}$. It has been indicated that following $2 \mathrm{~h}$ of milrinone withdrawal, the hemodynamic indexes and cardiac function indexes may be restored to the level prior to treatment, so the current recommendation is administration for $24 \mathrm{~h}$, which requires a maintenance dose to maintain the effective concentration at the loading dose after the vein, and the 

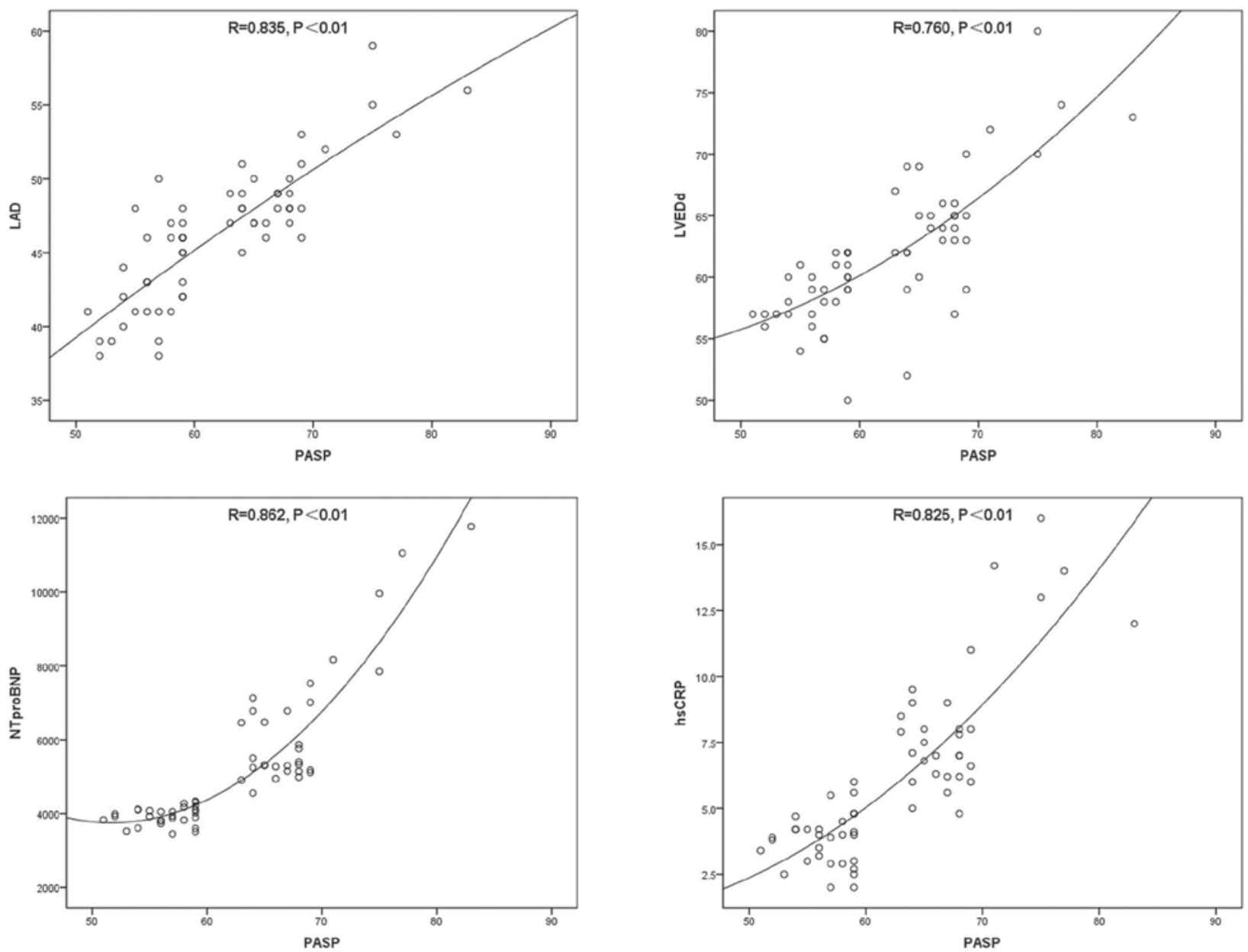

Figure 1. Scatter plots for the correlations between PASP+LAD $(\mathrm{R}=0.835, \mathrm{P}<0.01), \mathrm{PASP}+\mathrm{LVEDd}(\mathrm{R}=0.760, \mathrm{P}<0.01), \mathrm{PASP}+\mathrm{NT}-\mathrm{proBNP}(\mathrm{R}=0.862, \mathrm{P}<0.01)$ and PASP+hsCRP $(\mathrm{R}=0.825, \mathrm{P}<0.01)$ prior to therapy. PASP, pulmonary artery systolic pressure; LAD, left atrial diameter; LVEDd, left ventricle end diastolic diameter; NT-proBNP, N-terminal pro-B-type natriuretic peptide; hsCRP, hypersensitive C-reactive protein.

treatment efficacy is positively correlated with the dosage (19). Milrinone is a commonly used drug for the treatment of heart failure, and its effect on PH was also reported (3-6). Various studies have also assessed the inhalation of milrinone. In 2005, Lamarche et al (20) reported the effects of inhaled and intravenous milrinone on the function of pulmonary vascular endothelial cells in pigs following extracorporeal circulation. In 2012, Guo (3) reported that inhalation of milrinone significantly reduces the PAH and PVR, and increases the cardiac index in patients with severe left heart valvular disease, while the patients' HR and systemic vascular resistance were not significantly affected, which reflects good selectivity for pulmonary vessels.

The results of the present study indicate that conventional treatment improved the cardiac systolic function, but the improvement was not obvious. Instructions for the administration of milrinone currently only contain recommended doses for heart failure and not for PH. The dose chosen in the present study was in accordance with the dose reported for heart failure in the drug instructions, which describes a loading dose of 25-75 $\mu \mathrm{g} / \mathrm{kg}$ injected intravenously over 5-10 min and a maintenance dose of $0.25-1 \mu \mathrm{g} / \mathrm{kg} / \mathrm{min}$. A small dose of milrinone $(0.25 \mu \mathrm{g} / \mathrm{kg} / \mathrm{min})$ had no significant superiority compared with the conventional treatment group in improving
LV structure and LV systolic function. Following doubling the dose of milrinone to $0.5 \mu \mathrm{g} / \mathrm{kg} / \mathrm{min}, \mathrm{LV}$ structure and function are obviously improved compared with the conventional treatment group. The double dose of milrinone improved the left heart ventricular systolic function and structure compared with the small dose of milrinone, but the extent of improvement was not significant. The small dose $(0.25 \mu \mathrm{g} / \mathrm{kg} / \mathrm{min})$ and the double dose $(0.5 \mu \mathrm{g} / \mathrm{kg} / \mathrm{min})$ of milrinone significantly improved the left heart structure and LV systolic function in patients with mild and moderate PH-LHD. As there were only 2 patients with severe PH-LHD in group A and group $\mathrm{B}$, the number of samples was small and not suitable for comparison by statistical methods. The mechanism by which milrinone reduces LAD and LVEDd is as follows: Milrinone inhibits PDE III, increases cAMP in vascular smooth muscle cells, reduces the intracellular calcium concentration, inhibits excitatory contraction coupling, and dilates arteries and veins, which reduces the front and back load of the heart. The reduction in the volume of blood returning to the heart reduces the pressure in the heart and the inner diameter of the atrium and ventricle.

Right heart catheterization has been used to evaluate and diagnose PH. However, the tests are invasive, costly and use radioactivity, and it is therefore difficult to accept by 
Table VII. Correlation between PASP and LAD, LVEDd, LVEF, TAPSE, RVFAC, NT-proBNP and hsCRP prior to treatment.

\begin{tabular}{lcc}
\hline Parameter & $\mathrm{R}$ & P-value \\
\hline LAD & 0.835 & $<0.01$ \\
LVEDd & 0.760 & $<0.01$ \\
LVEF & -0.849 & $<0.01$ \\
TAPSE & -0.847 & $<0.01$ \\
RVFAC & -0.843 & $<0.01$ \\
NT-proBNP & 0.862 & $<0.01$ \\
hSCRP & 0.825 & $<0.01$ \\
\hline
\end{tabular}

LAD, left atrial diameter; LVEDd, left ventricle end diastolic diameter; LVEF, left ventricle ejection fraction; PASP, pulmonary artery systolic pressure; TAPSE, tricuspid annular plane systolic excursion; RVFAC, right ventricular fractional area change; NT-proBNP, N-terminal pro-B-type natriuretic peptide; hsCRP, hypersensitive C-reactive protein; $\mathrm{PH}$, pulmonary hypertension.

clinicians and patients. PASP may be estimated by tricuspid regurgitation velocity and exhibits a good correlation with pulmonary arterial pressure measured by right cardiac catheterization $(21,22)$. The present study demonstrates that milrinone significantly improves the PASP in patients with PH-LHD, while the improvement of the PASP was not obvious in the conventional treatment group. Milrinone significantly improved the PASP in patients with PH-LHD compared with that in the conventional treatment group, and the double dose of milrinone caused the PASP to further reduce. Milrinone significantly improved the PASP of patients with mild and moderate PH-LHD. In future studies, a statistical analysis should be performed by increasing the number of samples with severe PH-LHD.

According to the Pearson correlation analysis, PASP was strongly positively correlated with LAD, LVEDd, NT-proBNP and hsCRP, and strongly negatively correlated with LVEF, TAPSE and RVFAC. Therefore, the severity of PH in PH-LHD patients may be estimated according to the above indexes.

The present study demonstrated that conventional treatment does not significantly improve the right ventricular function. Milrinone significantly improved the right heart function of PH-LHD patients compared with the conventional treatment. The small dose of milrinone $(0.25 \mu \mathrm{g} / \mathrm{kg} / \mathrm{min})$ and the double dose of milrinone $(0.5 \mu \mathrm{g} / \mathrm{kg} / \mathrm{min})$ significantly improved the right heart function. Milrinone significantly improved the right heart function in in patients with mild and moderate HP-LHD. However, in the routine treatment group, TAPSE was significantly improved but RVFAC has not significantly changed following treatment in patients with mild and moderate HP-LHD. Multiple measures, including three-dimensional echocardiography and cardiac magnetic resonance, will be used to assess whether the right ventricular function may be significantly improved in future research for patients with PH-LHD. Since there were only 2 patients with severe PH-LHD in group A or group B, and 1 patient with severe $\mathrm{PH}-\mathrm{LHD}$ in group $\mathrm{C}$, statistical analysis may be performed in future studies by increasing the number of samples.

The latest guidelines for PH of The European Heart Association from 2015 recommended BNP as the only biomarker for PAH risk assessment (23). NT-proBNP has a good prognostic value in pediatric and adult patients with PAH (24). Andreassen et al (25) confirmed that the elevated levels of NT-proBNP in PAH patients are associated with hemodynamics. Kolditz et al (26) suggested that different biomarkers respond to different aspects of the pathogenesis of PH and NT-proBNP was significantly correlated with hemodynamics. The present study indicated that milrinone significantly reduces NT-proBNP and hsCRP in patients with PH-LHD. In the routine treatment group C, NT-proBNP was significantly decreased, while the decrease of hsCRP was not significant. The small dose $(0.25 \mu \mathrm{g} / \mathrm{kg} / \mathrm{min})$ and the double dose $(0.5 \mu \mathrm{g} / \mathrm{kg} / \mathrm{min})$ of milrinone significantly decreased NT-proBNP and hsCRP compared with those in the conventional treatment group. However, by comparing the results of group A and B following treatment, it was revealed that following increasing the dose of milrinone, the decrease of NT-proBNP and hsCRP was not significant. In future studies, the dose of milrinone may be further increased to observe whether these indexes may be further reduced. The present study indicated that the small dose $(0.25 \mu \mathrm{g} / \mathrm{kg} / \mathrm{min})$ and the double dose $(0.5 \mu \mathrm{g} / \mathrm{kg} / \mathrm{min})$ of milrinone significantly decrease NT-proBNP and hsCRP in patients with mild and moderate PH-LHD. The present study suggested that PASP was strongly positively correlated with NT-proBNP and hsCRP $(\mathrm{P}<0.01)$. The degree of pulmonary artery pressure, and the degree of improvement may be evaluated according to the NT-proBNP and hsCRP. The following mechanism of NT-proBNP reduction following the application of milrinone is suggested: i) Milrinone significantly enhances cardiac contractility, increases the ejection fraction, increases cardiac output per beat, and reduces the volume load of the ventricle, thus reducing the secretion of NT-proBNP. ii) Milrinone improves diastolic ventricular compliance and ventricular diastolic function, so that the LV end-diastolic filling pressure is reduced, thus reducing the secretion of NT-proBNP.

In the present study, 4 cases of hypotension occurred when patients were given an intravenous milrinone load dose (BP $<90 / 60 \mathrm{mmHg}$ ). Following intravenous infusion of dopamine, the BP rose to $>90 / 60 \mathrm{mmHg}$. The adverse effects of fatigue and HR increase also occurred when the load dose was given, but the symptoms disappeared following the maintenance dose was given. No adverse effects of arrhythmia and thrombocytopenia were encountered in the present study. Milrinone has been reported to affect platelets in a dose-dependent manner (27), and Kikura et al (28) demonstrated that long-term oral administration of amrinone caused thrombocytopenia; however, the short-term use of milrinone had no significant effect on platelet count and function.

The major causative factors of adverse reactions to milrinone are as follows: i) Pharmacological action. Competitive inhibition of PDE III by milrinone increases cGMP in vascular smooth muscle cells and reduces the BP (12). ii) Pathological factors. Based on the findings of the current study, milrinone opens the calcium channel in the myocardial cell membrane during myocardial depolarization and the influx of calcium ions 
Table VIII. Comparison of adverse reactions in the three groups of patients with pulmonary hypertension due to left heart disease, $\mathrm{n}(\%)$.

\begin{tabular}{|c|c|c|c|}
\hline & Group A $(n=20)$ & Group B $(n=20)$ & Group C $(\mathrm{n}=20)$ \\
\hline Adverse reaction & Cases incidence $(\%)$ & Cases incidence $(\%)$ & Cases incidence $(\%)$ \\
\hline Headache & $2(10)$ & $1(5)$ & $1(5)$ \\
\hline Asthenia & $2(10)$ & $2(10)$ & $1(5)$ \\
\hline Increased heat rate & $1(5)$ & $1(5)$ & $1(5)$ \\
\hline Hypopiesia & $1(5)$ & $3(15)$ & 0 \\
\hline
\end{tabular}
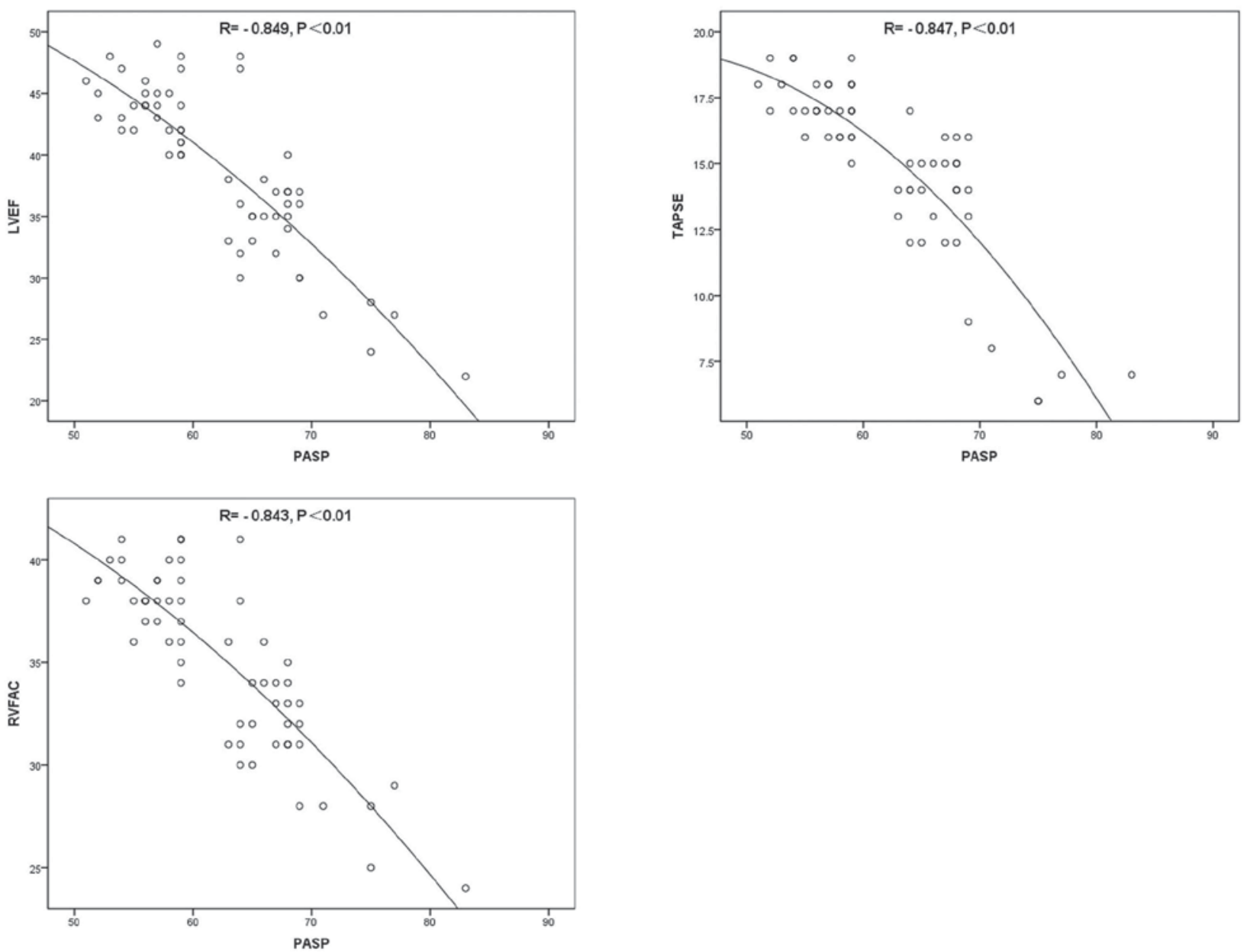

Figure 2. Scatter plots for the correlation between PASP+LVEF ( $R=-0.849, \mathrm{P}<0.01), \mathrm{PASP}+\mathrm{TAPSE}(\mathrm{R}=-0.847, \mathrm{P}<0.01)$ and $\mathrm{PASP}+\mathrm{RVFAC}(\mathrm{R}=-0.843, \mathrm{P}<0.01)$ prior to therapy. PASP, pulmonary artery systolic pressure; LVEF, left ventricle ejection fraction; TAPSE, tricuspid annular plane systolic excursion; RVFAC, right ventricular fractional area change.

increases the concentration of calcium ions in the cells. The rate of depolarization of sinus node pacemaker cells was accelerated during the diastolic phase. As a result, the P-R interval is shortened and the atrioventricular node conduction is accelerated. Milrinone increases the intracellular calcium concentration by increasing the concentration of protein kinase A (PKA) (12). PKA also further activates the slow potassium channel and L-type calcium channel in the heart, which in turn shortens the refractory period of the atrium (12). The adverse effects of milrinone on long-term use are arrhythmia, such as atrial fibrillation and frequent ventricular premature beats, so it should be used for a short time (19). iii) Drug concentration. Based on current research, when a single dose is too large or the intravenous drip is too fast, this may lead to high local concentrations in the body in a short period of time, which may cause adverse reactions. iv) Combination of milrinone with potent diuretics. As heart failure is a complex clinical syndrome, it frequently requires a combination of multiple drugs. In the present study, 4 cases of hypotension occurred following the use of milrinone on the basis of strong diuretics. It was suggested that the vasodilator effect of milrinone was enhanced upon decreasing the blood volume, resulting in a sharp decrease in BP. 
In the present study, the maintenance dose of milrinone did not cause any significant adverse reactions during the course of the treatment. The results of Baruch et al (29) demonstrated that if no load, at $25-75 \mu \mathrm{g} / \mathrm{kg}$ intravenously over 5-10 min, was administered in advance and milrinone was injected over $30 \mathrm{~min}$ at $0.5 \mu \mathrm{g} / \mathrm{kg} / \mathrm{min}$, a hemodynamic effect is evident. The PCWP was similar to that of application load following $2 \mathrm{~h}$ of continuous infusion and cardiac index and plasma concentration of milrinone were similar following $3 \mathrm{~h}$ of continuous infusion (29). However, 3 cases of hypotension and 2 cases of arrhythmia were observed in the load dose group, which was associated to the short injection time and fast injection speed (29). A previous study suggested that if milrinone is not administered at a load dose, it may not affect clinical outcomes and reduce adverse events (29). Through injection via a micropump, the input speed of the drug may be accurately controlled, and at the same time, the dose may be adjusted according to the hemodynamic changes of the patients to avoid large increases in the local concentration within a short period of time. Due to the short plasma half-life of milrinone, $80 \%$ of the drug is excreted by the kidneys following $24 \mathrm{~h}$ of treatment. It has been reported that following milrinone withdrawal for $2 \mathrm{~h}$, the hemodynamic and cardiac function indices were restored to the level of that prior to treatment; therefore, it is recommended that the drug be administered over $24 \mathrm{~h}$ (30). Following the loading dose, the maintenance dose must be administered by infusion to maintain an effective plasma concentration, and a positive correlation between the dosage and efficacy has been reported (30). Monitoring should be performed during the course of medication, and the dynamic changes of BP, HR and other clinical symptoms must be closely monitored; first aid should be provided immediately in case of hypotension. If atrial flutter/atrial fibrillation occurs, milrinone may in theory slightly increase atrioventricular node conduction and the ventricular rate. Therefore, it is suggested that digitalis preparation is used to control the ventricular rate in advance.

Based on the present results, the following may be concluded: i) Milrinone reduces PASP in patients with PH-LHD, and improves the cardiac structure, cardiac function and biochemical indexes; ii) a small dose of milrinone may significantly reduce PASP in patients with mild and moderate PH-LHD, and significantly improve the cardiac structure, cardiac function and biochemical indexes; iii) increasing the dose of milrinone may further improve PASP in patients with mild and moderate PH-LHD, but the cardiac structure and cardiac function are not significantly improved in comparison with low-dose milrinone; iv) PASP was positively correlated with LAD, LVEDd, NT-proBNP and hsCRP, and negatively correlated with LVEF, TAPSE and RVFAC; v) the maintenance dose of milrinone did not cause any significant adverse reactions during the course of the treatment. Therefore, it needs to be investigated whether the use of milrinone with no load dose may reduce adverse reactions without affecting its clinical efficacy.

In the present study, the number of patients with severe PH-LHD was small, so that no statistical analysis could be performed, and further studies are required to increase the number of patients with severe PH-LHD to provide meaningful clinical results. In the present study, only PASP was used to assess PAH. In future studies, ultrasound and right heart catheterization may be combined to evaluate $\mathrm{PAH}$ and increase the accuracy of its determination. In future studies, the milrinone maintenance dose may be increased within the specified dose range, to see if heart function and structure, PASP and test indicators of patients with PH-LHD may be improved, and whether adverse events increase with higher doses.

\section{Acknowledgements}

Not applicable.

\section{Funding}

No funding was received.

\section{Availability of data and materials}

The datasets used and/or analyzed during the current study are available from the corresponding author on reasonable request.

\section{Authors' contributions}

$\mathrm{BH}$ contributed to the conception and design of the study, the acquisition, analysis and interpretation of data and drafting and revising of the manuscript. QW contributed to the acquisition, analysis and interpretation of data. The final version of the manuscript has been read and approved by all authors.

\section{Ethical approval and consent to participate}

All of the participants of this study provided written informed consent and volunteered to join the study. The study was approved by the Ethics Committee of The Sixth People's Hospital of Jinan (Jinan, China).

\section{Consent for publication}

Not applicable.

\section{Competing interests}

The authors declare that they have no competing interests.

\section{References}

1. Bursi F, McNallan SM, Redfield MM, Nkomo VT, Lam CS, Weston SA, Jiang R and Roger VL: Pulmonary pressures and death in heart failure: A community study. J Am Coll Cardiol 59: 222-231. 2012.

2. Barst RJ, Ertel SI, Beghetti M and Ivy DD: Pulmonary arterial hypertension: A comparison between children and adults. Eur Respir J 37: 665-677, 2011.

3. Guo H: Applied research of specific phosphodiesterase inhibitor during perioperative period of rheumatic valvular disease combining with pulmonary hypertension (unpublished $\mathrm{PhD}$ thesis). Southern Medical University, 2012.

4. Yu Y and Shi J: Effect of milrinone on pulmonary function and dynamics of congenital heart disease with pulmonary hypertension in patients with pulmonary oxygen. J Practical Med 28: 469-470, 2012.

5. Xu D: Effect of milrinone on pulmonary hypertension after cardiac surgery. Guide Chin Med 11: 200-202, 2013 (In Chinese). 
6. Yao B, Da G, Li Y, Wu J, Li Z, Yang Y, Zhu J, Wang W and Yang J: The protective effect of milrinone on heart function in plateau region in children with congenital heart disease and pulmonary hypertension after operation. Shandong Med J 54: 49-51, 2014 (In Chinese).

7. Galiè N, Hoeper MM, Humbert M, Torbicki A, Vachiery JL, Barbera JA, Beghetti M, Corris P, Gaine S, Gibbs JS, et al: Guidelines for the diagnosis and treatment of pulmonary hypertension: The Task force for the diagnosis and treatment of pulmonary hypertension of the European society of cardiology (ESC) and the European Respiratory Society (ERS), endorsed by the International society of heart and lung transplantation (ISHLT). Eur Heart J 30: 2493-2537, 2009.

8. Yock PG and Popp RL: Noninvasive estimation of right ventricular systolic pressure by Doppler ultrasound in patients with tricuspid regurgitation. Circulation 70: 657-662, 1984.

9. Trammell AW, Pugh ME, Newman JH, Hemnes AR and Robbins IM: Use of pulmonary arterial hypertension-approved therapy in the treatment of non-group 1 pulmonary hypertension at US referral centers. Pulm Circ 5: 356-363, 2015.

10. Li A, Xue C, Song M, MA S and Jia W: The clinical efficacy and safety of oral administration of atorvastatin in patients with pulmonary hypertension with left heart disease. Ningxia Med J 37: 319-321, 2015 (In Chinese).

11. Lian X, Yang G and Wang E: Efficacy of levosimendan combined with sildenafil in the treatment of chronic heart failure with pulmonary hypertension. Practical J 40: 14-16, 2013.

12. Shipley JB, Tolman D, Hastillo A and Hess ML: Milrilone: Basic and clinical pharmacology and acute and chronic management Am J Med Sci 311: 286-291, 1996.

13. Schudt C, Winder S, Forderkunz S, Hatzelmann A and Ullrich V: Influence of selective phosphodiesterase inhibitors on human neutrophil functions and levels of cAMP and Cai. Naunyn Schmiedebergs Arch Pharmacol 344: 682-690, 1991.

14. Kume M, Banafsche R, Yamamoto Y, Yamaoka Y, Nobiling R, Gebhard MM and Klar E: Dynamic changes of post-ischemic hepatic microcirculation improved by a pre-treatment of phosphodiesterase-3 inhibitor, milrinone. J Surg Res 136: 209-218, 2006

15. Endres S, Sinba B and Fulle HJ: Amrinone suppresses the synthesis of tumor necrosis factor-alpha in human mononuclear cells. Shock 1: 377-380, 1994.

16. Kawamura A, Yoshikawa $\mathrm{T}$, Takahashi $\mathrm{T}$, Hayashi $\mathrm{T}$, Takahashi E, Anzai T, Sato T and Ogawa S: Randomized trial of phosphodiesterase inhibitors versus catecholamines in patient with acutely decompensated heart failure. Jpn Circ J 65: 858-862, 2001.

17. Lanfear DE, Hasan R, Gupta RC, Williams C, Czerska B, Tita C, Bazari R and Sabbah HN: Short term effects of milrinone on biomarkers of necrosis, apoptosis, and inflammation in patients with severe heart failure. J Transl Med 29: 67, 2009.

18. Siflinger-Bimboim A, Bode DC and Malik AB: Adenosine 3',5'-cyclic monophosphate attenuates neutrophil-mediated increase in endothelial permeability. Am J Physiol 264: H370-H375, 1993

19. Fleming GA, Murray KT, Yu C, Byrne JG, Greelish JP, Petracek MR, Hoff SJ, Ball SK, Brown NJ and Pretorius M: Milrinone use is associated with post-operative atrial fibrillation after cardiac surgery. Circulation 118: 1619-1625, 2008.
20. Lamarche Y, Malo O, Thorin E, Denault A, Carrier M, Roy J and Perrault LP: Inhaled but not intravenous milrinone prevents pulmonary endothelial dysfunction after cardiopumonary bypass. J Thorac Cardiovasc Surg 130: 83-92, 2005.

21. Burgess MI, Mogulkoc N, Bright-Thomas RJ, Bishop P, Egan JJ and Ray SG: Comparison of echocardiographic markers of right ventricular function in determining prognosis in chronic pulmonary disease. J Am Soc Echocardiogr 15: 633-639, 2002.

22. Li Y, Wang Y, Zhai Z, Guo X, Yang Y and Lu X: Real-time three-dimensional echocardiography to assess right ventricle function in patients with pulmonary hypertension. PLoS One 10: e0129557, 2015.

23. Galiè N, Humbert M, Vachiery JL, Gibbs S, Lang I, Torbicki A, Simonneau G, Peacock A, Vonk Noordegraaf A, Beghetti M, et al: 2015 ESC/ERS Guidelines for the diagnosis and treatment of pulmonary hypertension: The joint task force for the diagnosis and treatment of pulmonary hypertension of the European Society of Cardiology (ESC) and the European Respiratory Society (ERS): Endorsed by: Association for European Paediatric and Congenital Cardiology (AEPC), International Society for Heart and Lung Transplantation (ISHLT). Eur Heart J 37: 67-119, 2016.

24. Harbaum L, Hennigs JK, Baumann HJ, Lüneburg N, Griesch E, Bokemeyer C, Grünig E and Klose $\mathrm{H}$ : N-terminal pro-brain natriuretic peptide is a useful prognostic marker in patients with pre-capillary pulmonary hypertension and renal insufficiency. PLoS One 9: e94263, 2014.

25. Andreassen AK, Wergeland R, Simonsen S, Geiran O, Guevara C and Ueland T: N-terminal pro-B-type natriuretic peptide as an indicator of disease severity in a heterogeneous group of patients with chronic precapillary pulmonary hypertension. Am J Cardiol 98: 525-529, 2006.

26. Kolditz M, Seyfarth HJ, Wilkens H, Ewert R, Bollmann T, Dinter C, Hertel S, Klose H, Opitz C, Grünig E, et al: MR-proADM predicts exercise capacity and survival superior to other biomarkers in PH. Lung 193: 901-910, 2015.

27. Wesley MC, McGowan FX, Castro RA, Dissanayake S, Zurakowski D and Dinardo JA: The effect of milrinone on platelet activation as determined by TEG platelet mapping. Anesth Analg 108: 1425-1429, 2009.

28. Kikura M, Lee MK, Safon RA, Bailey JM and Levy JH: The effects of milrinone on platelets in patients undergoing cardiac surgery. Anesth Analg 81: 44-48, 1995.

29. Baruch L, Patacsil P, Hameed A, Pina I and Loh E: Pharmacodynamic effects of milrinone with and without a bolus loading infusion. Am Heart J 141: 266-273, 2001.

30. Huang LY and Yang Y: Clinical study of milrinone in the treatment of neonatal persistent pulmonary hypertension. Straits Sci 8: 111-113, 2012 (In Chinese).

This work is licensed under a Creative Commons Attribution 4.0 International (CC BY-NC 4.0) License 